\title{
Forecast for the second Covid-19 wave based on the improved SIR model with a constant ratio of recovery to infection rate
}

\author{
M. Kröger ${ }^{1, *}$, R. Schlickeiser ${ }^{2,3, * *}$ \\ ${ }^{1}$ Polymer Physics, Department of Materials, ETH Zurich, \\ Zurich CH-8093, Switzerland, ORCID 0000-0003-1402-671 \\ 2 Institut für Theoretische Physik, Lehrstuhl IV: Weltraum- und Astrophysik, \\ Ruhr-Universität Bochum, D-44780 Bochum, Germany, ORCID 0000-0003-3171-5079 \\ ${ }^{3}$ Institut für Theoretische Physik und Astrophysik, Christian-Albrechts-Universität zu Kiel, Leibnizstr. 15, D-24118 Kiel, Germany
}

(Dated: January 16, 2021)

\begin{abstract}
We start out by deriving simple analytic expressions for all measurable amounts of cases and fatalities during a pandemic evolution exhibiting multiple waves, described by the semi-time SIR model. The approximant shares all relevant features with the exact solution, including time and position of the peak of daily new infections, as well as the asymptotic behaviors at small and large times. We derive exact analytic expressions for the early doubling time, late half decay time, and a half-early peak law, characterizing the dynamical evolution. We show, in particular, how the asymmetry of the first epidemic wave and its exponential tails are affected by the initial conditions; a feature that has no analogue in the all-time SIR model. We apply the approach to available data from different continents. Our analysis reveals that the immunity is very strongly increasing during the 2nd wave, while it was still at a very moderate level of a few percent in several countries at the end of the first wave. The wave-specific SIR parameters describing the infection and recovery rates we find to behave in a similar fashion, while their ratio $k$ was decreasing only by a about $5 \%$ for most countries. Still, an apparently moderate change of $k$ can have significant consequences for the relevant numbers like the final amount of infected or deceased population. As we have shown, the probability for an additional wave is however low in several countries due to the fraction of immune inhabitants at the end of the 2nd wave, irrespective the currently ongoing vaccination efforts. We compare with alternate approaches.
\end{abstract}

Keywords: coronavirus; statistical analysis; extrapolation; parameter estimation; pandemic spreading

\section{INTRODUCTION}

Currently, many countries over the world have to cope with handling the subsequent (second, third...) wave outbreaks of Covid-19 pandemic. The onset of a subsequent wave is most probably caused by a sudden mutation of the virus resulting in an effective (about 40 percent) increase of the infection rate. Of high medical and economic interest are reliable predictions on the duration, peak days and total amplitudes of both the number of infections and the number of fatalities which are closely related to the maximum need of breathing apparati in hospitals in order not to overburden the medical clinics capacities and to avoid fateful triage decisions.

In the past reasonably accurate (within 50 percent) predictions on the first Covid-19 wave have been made ${ }^{1-4}$ adopting the Gaussian distribution for the daily rate of new cases and the corresponding cumulative numbers (including both infections and fatalities) for many countries in the world with well monitored case rates. By modeling simultaneously both infection and fatality rates the dark number of infections and the degree of herd immunity from the first wave has been determined in several countries ${ }^{5}$ by adopting a mortality rate of 0.5 percent, i.e., one out of 200 infected persons ultimately dies from Covid-19. While the Gauss distribution can be justified both from the central limit theorem of statistics ${ }^{1}$

*rsch@tp4.rub.de, mk@mat.etz.ch and for early times until the peak time from the susceptibleinfectious-recovered/removed (SIR) epidemic model, ${ }^{6-9}$ it is less accurate at late times of the wave evolution as compared to monitored data. ${ }^{10}$

The SIR model ${ }^{6,7}$ describes the time evolution of infectious diseases in human populations, and is the simplest and most fundamental of the compartmental models and its variations. It had been solved numerically using various approaches, including Monte Carlo methods, wavelets, fuzzy control, deep learning etc. ${ }^{11-31}$ and approximate solutions had been proposed. ${ }^{32,33}$ Our recent work ${ }^{34,35}$ improved the analytical modelling of epidemics based on the well-established SIR epidemic model invented nearly 100 years ago.

There are two variants of implementing the SIR model, as described in detail previously. ${ }^{34,35}$ The all-time SIR model must use an initial condition that is compatible with the SIR equations of change, and then allows to 'predict' both the past and present consistently. The semi-time SIR model can be used with any initial condition, but makes predictions only about the future. Often the 2 nd variant is used without noticing the inconsistency, while the inconsistency had already been noted by Kendall. ${ }^{6}$ Assuming a constant ratio between infection and recovery rates, the all-time SIR model does predict a single wave only, while the semi-time SIR model we are going to use in this work allows to model many waves with their own specific parameters, that capture only the future development in each case.

For both variants the considered population of $N \gg 1$ per- 
sons is assigned to the three compartments $s$ (susceptible), $i$ (infectious), or $r$ (recovered/removed). Persons from the population may progress with time between these compartments with given infection $(a(t))$ and recovery rates $(\mu(t))$ which in general vary differently with time. As demonstrated before ${ }^{34}$ it is convenient to introduce with $I(t)=i(t) / N, S(t)=$ $s(t) / N$ and $R(t)=r(t) / N$ the infected, susceptible and recovered/removed fractions of persons involved in the infection at time $t$, with the sum requirement $I(t)+S(t)+R(t)=1$. Moreover, if $t_{0, n}$ denotes starting time of the $n$-th wave with the initial conditions $I\left(t_{0, n}\right)=\eta$ and $S\left(t_{0 . n}\right)=1-\eta$, it is also appropriate to introduce the dimensionless reduced time variable

$$
\tau(t)=\int_{t_{0, n}}^{t} d \xi a(\xi)
$$

accounting for arbitrary but given time-dependent infection rates $a(t)$. For the special and important case of a timeindependent ratio $K(t)=\mu(t) / a(t)=k=$ const., analytical results of the SIR model have been recently derived ${ }^{34,35}$ both for the all-time and semi-time cases appropriate for the first and subsequent wave evolution, respectively. For a growing epidemics outburst, exhibiting a peak at some time past $t_{0, n}$ in the future, the constant ratio $k$ has to be smaller than $1-2 \eta$, where as noted before $\eta=I\left(t_{0, n}\right)$ denotes the infected fraction at the starting time $t_{0, n}$ of the $n$-th wave. The requirement $k<1-2 \eta$ corresponds to the initial infection rate $a_{0}$ at time $t_{0, n}$ being larger than the initial recovery rate $\mu_{0}$, both in units of days ${ }^{-1}$. Alternatively, for initial ratios $k>1-2 \eta$ the wave amplitude is purely decaying from its maximum at $t_{0, n}$ without exhibiting a wave phenomenon. ${ }^{35}$ Here the time $t_{0 . n}$ refers to the observing time when the onset of a new $n$-th wave is recognized by the monitoring of case rates.

It has been demonstrated ${ }^{35}$ that the two parameters $k$ and $\eta$ determine the full evolution of each subsequent waves in reduced time (1). Moreover, it is important to emphasize that in the peak case where $k<1-2 \eta$ the SIR model not only provides a causal connection between the early (before the peak) and late time development of the pandemic wave, but that the parameters $k$ and $\eta$ obtained from fitting the early time evolution also determine the final and maximum values of the cumulative and daily case rates.

Below, we will derive simple analytic expressions for all measurable amounts of cases and fatalities during a pandemic evolution described by the semi-time SIR model, that share all relevant features with the exact solution of the semi-time SIR model, including time and position of the peak of daily new infections, as well as the asymptotic behaviors at small and large times. The expressions are so precise that they can be used instead of a numerical solution of the SIR model. The advantage of an analytical expression is obvious, as it allows to quickly determine the SIR parameters from the measured data well ahead the peak time, and thus allows for predictions that serve as a prerequisite to make decisions. We apply the approach to eight different countries from different continents. We begin by summarizing the exact features of the semi-time SIR model, stating the approximants for the reliably measurable quantities, and collect all the derivations of the new ap- proximant in an appendix.

\section{SIR-MODEL RESULTS}

\section{A. Exact results}

The exact solution of the SIR model with a constant ratio of the recovery to infection rate in the semi-time case ${ }^{35}$ (hereafter referred to as KS-SIR model) is given by

$$
\begin{aligned}
\tau & =\int_{\eta}^{J} \frac{d y}{N(y)}, \\
N(y) & =(1-y)[y+k \varepsilon+k \ln (1-y)]
\end{aligned}
$$

with $\eta=1-e^{-\varepsilon}, \tau$ given by Eq. (1), and $J=1-S$ denoting the cumulative fraction of new cases. Differentiating Eq. (2a) with respect to $\tau$ yields for the corresponding rate of new cases

$$
j(\tau)=\frac{d J(\tau)}{d \tau}=(1-J)[J+k \varepsilon+k \ln (1-J)]=N(J)
$$

As shown before ${ }^{35}$ without explicit inversion of Eq. (2), Eqs. (2) and (3) yield for the final cumulative number fraction of infected persons $J_{\infty}=\lim _{\tau \rightarrow \infty} J(\tau)$ and for the maximum rate of new infections $j_{\max }$ occuring at $J_{0}$ that

$$
\begin{aligned}
J_{\infty} & =1+k W_{0}(\alpha), \quad \alpha=-\frac{(1-\eta) e^{-1 / k}}{k} \\
J_{0} & =1+\frac{k}{2} W_{-1}\left(\alpha_{0}\right), \quad \alpha_{0}=\frac{2 \alpha}{e} \\
j_{\max } & =N\left(J_{0}\right)=\left(1-J_{0}\right)\left(1-J_{0}-k\right) \\
& =\frac{k^{2}}{4}\left(\left[1+W_{-1}\left(\alpha_{0}\right)\right]^{2}-1\right)
\end{aligned}
$$

in terms of the principal $\left(W_{0}\right)$ and non-principal $\left(W_{-1}\right)$ solutions of Lambert's equation, ${ }^{34}$ the well-known Lambert's functions. The asymmetry of $j(\tau)$ about its peak time is encoded in a difference between $J_{0}$ and $J_{\infty} / 2$.

\section{B. Constrained 2nd order polynomial approximation}

We approximately invert the solution (2) by using the constrained Taylor-expansions of the reciprocal integrand

$$
\begin{aligned}
N(y) & \simeq j_{c}(y)=\sum_{i=0}^{2} c_{i}(y-\eta)^{i} \\
& =c_{0}+c_{1}(y-\eta)+c_{2}(y-\eta)^{2} \\
N(y) & \simeq j_{d}(y)=\sum_{i=1}^{2} d_{i}\left(J_{\infty}-y\right)^{i} \\
& =d_{1}\left(J_{\infty}-y\right)+d_{2}\left(J_{\infty}-y\right)^{2}
\end{aligned}
$$

about $y_{c}=\eta$ and $y_{d}=J_{\infty}$ up to 2 nd order, respectively. Here $c_{0}, c_{1}$ and $d_{1}$ are the respectively positively-valued Taylor ex- 
pansion coefficients given by

$$
\begin{aligned}
& c_{0}=(1-\eta) \eta \\
& c_{1}=1-k-2 \eta \\
& d_{1}=J_{\infty}-(1-k)
\end{aligned}
$$

Hereby, constrained expansion refers to chosing the secondorder expansion coefficients $c_{2}$ and $d_{2}$ such that the respective expansion evaluated at $y=J_{0}$ yield the maximum value of the daily case rate i.e.

$$
j_{c}\left(y=J_{0}\right)=j_{d}\left(y=J_{0}\right)=j_{\max }
$$

with $j_{\max }$ from Eq. (7). The requirement (13) then readily provides

$$
\begin{aligned}
c_{2} & =\frac{j_{\max }-c_{0}-c_{1}\left(J_{0}-\eta\right)}{\left(J_{0}-\eta\right)^{2}} \\
d_{2} & =\frac{j_{\max }-d_{1}\left(J_{\infty}-J_{0}\right)}{\left(J_{\infty}-J_{0}\right)^{2}}
\end{aligned}
$$

While $c_{2}$ is negative for all $k<1-2 \eta$, the coefficient $d_{2}$ may have either sign. It is important to realize that all quantities (4) - (7) and coefficients (10) -(12) as well as (14) -(15) are solely determined by the two basic KS-SIR model parameters $k$ and $\eta$.

According to Eqs. (8) - (9) we have thus constructed the approximation for the daily rate $j(J)$ as a function of the cumulative number

$$
j(J) \simeq \begin{cases}c_{0}+c_{1}(J-\eta)+c_{2}(J-\eta)^{2} & \text { for } J \leq J_{0} \\ d_{1}\left(J_{\infty}-J\right)+d_{2}\left(J_{\infty}-J\right)^{2} & \text { for } J \geq J_{0},\end{cases}
$$

which is continuous at $J=J_{0}$ where $j\left(J_{0}\right)=j_{\max }$ attains its peak value.

As we show next, inserting the approximation (16) then allows the analytical inversion of the solution (2) providing $J(\tau)$ as a function of the reduced time $\tau$ which then can be used either in Eq. (16) or Eq. (3) to infer also the rate $j(\tau)$ as a function of reduced time. The remaining SIR quantities are then obtained from $J(\tau)$ as well via $S(\tau)=1-J(\tau), I(\tau)=J(\tau)+k \varepsilon+k \ln [1-J(\tau)]$ and $R(\tau)=1-S(\tau)-I(\tau)=-k[\varepsilon+\ln (1-J(\tau))]$.

\section{Cumulative number and rate by exact inversion of the approximant}

While we have expressed $\tau$ in terms of $J$ above, for all practical purposes one is interested in the reverse relationship, the time $\tau$-dependent behavior of $J$ and also $j$. With the approximations (8) - (9) we obtain for the solution (2)

$$
\tau \simeq \begin{cases}\int_{\eta}^{J} \frac{d y}{j_{c}(y)} & \text { for } J \leq J_{0} \\ \int_{\eta}^{J_{0}} \frac{d y}{j_{c}(y)}+\int_{J_{0}}^{J} \frac{d y}{j_{d}(y)} & \text { for } J \geq J_{0}\end{cases}
$$

Introducing the peak time scale

$$
\tau_{m}=\int_{\eta}^{J_{0}} \frac{d y}{j_{c}(y)}
$$

corresponding to $J=J_{0}$ we may write Eq. (17) as

$$
\tau \simeq \begin{cases}\tau_{m}-\int_{J}^{J_{0}} \frac{d y}{j_{c}(y)} & \text { for } \tau \leq \tau_{m} \\ \tau_{m}+\int_{J_{0}}^{J} \frac{d y}{j_{d}(y)} & \text { for } \tau \geq \tau_{m}\end{cases}
$$

In Appendix A the integrals appearing in Eqs. (18) and (19) are calculated leading to

$$
J(\tau) \simeq \begin{cases}\eta+\frac{J_{0}-\eta}{1+\sqrt{\frac{j_{\max }}{c_{0}} \frac{\sinh \left[c_{3}\left(\tau_{m}-\tau\right]\right.}{\sinh \left(c_{3} \tau\right)}}} & \text { for } \tau \leq \tau_{m} \\ J_{\infty}-\frac{j_{\max }}{d_{1}\left(J_{\infty}-J_{0}\right)}\left[e^{d_{1}\left(\tau_{0}-\tau_{m}\right)}-1\right]+1 & \text { for } \tau \geq \tau_{m}\end{cases}
$$

with the dimensionless peak time

$$
\tau_{m}=\frac{1}{c_{3}} \operatorname{artanh} \frac{2 c_{3}}{c_{1}+\frac{2 c_{0}}{J_{0}-\eta}},
$$

and the abbreviation

$$
c_{3}=\sqrt{\left(c_{1} / 2\right)^{2}-c_{0} c_{2}}
$$

Note that Eq. (20) obviously exhibits the correct extremal behaviors, $J(0)=\eta, J\left(\tau_{m}\right)=J_{0}$, and $\lim _{\tau \rightarrow \infty} J(\tau)=J_{\infty}$.

Inserting the cumulative number $J(\tau)$ from Eq. (20) into Eq. (16), or alternatively, from $d J / d \tau$, we obtain for the time dependence of the corresponding reduced rate (16)

$\frac{j(\tau)}{j_{\max }}= \begin{cases}\left(\frac{\sinh \left(c_{3} \tau_{m}\right)}{\sinh \left(c_{3} \tau\right)+\sqrt{\frac{j_{\max }}{c_{0}}} \sinh \left[c_{3}\left(\tau_{m}-\tau\right)\right]}\right)^{2} & \text { for } \tau \leq \tau_{m} \\ \frac{e^{d_{1}\left(\tau-\tau_{m}\right)}}{\left(1+\frac{j_{\max }}{d_{1}\left(J_{\infty}-J_{0}\right)}\left[e^{d_{1}\left(\tau-\tau_{m}\right)}-1\right]\right)^{2}} & \text { for } \tau \geq \tau_{m}\end{cases}$

The solutions (23) correctly reduce to $j_{\max }$ for $\tau=\tau_{m}$. Moreover, $j(0)=c_{0}=\eta(1-\eta)$ and $\lim _{\tau \rightarrow \infty} j(\tau)=0$ are obviously correctly captured. Details of the calculations leading to Eqs. (23) are also collected in Appendix A. The relative errors of the approximants are reported in Appendix C.

\section{Early and late reduced time evolution}

For very late reduced time $\tau \gg \tau_{m}$ the rate $j(\tau)$, second case in Eq. (23), approaches the decreasing exponential function in reduced time

$$
\begin{aligned}
j\left(\tau \gg \tau_{m}\right) & \simeq \frac{d_{1}^{2}\left(J_{\infty}-J_{0}\right)^{2}}{j_{\max }} e^{-d_{1}\left(\tau-\tau_{m}\right)} \\
& =\frac{\left[J_{\infty}-(1-k)\right]^{2}\left(J_{\infty}-j_{0}\right)^{2}}{\left(1-J_{0}\right)\left(1-k-J_{0}\right)} e^{-\left[J_{\infty}-(1-k)\right]\left(\tau-\tau_{m}\right)}
\end{aligned}
$$

with the decay half-reduced time ${ }^{36}$

$$
\tau_{1 / 2}=\frac{\ln 2}{J_{\infty}-(1-k)} \approx \frac{0.693}{J_{\infty}-(1-k)}
$$

defined by $j\left(\tau+\tau_{1 / 2}\right)=j(\tau) / 2$. Similarly, for very early reduced time $\tau \ll \tau_{m}$, provided such regime exists, as its 
pronounced appearance depends on the values for $\eta$ and $k$, the rate $j(\tau)$, first case in Eq. (23), is an increasing exponential function in reduced time with the doubling time

$$
\tau_{2}=\frac{\ln \sqrt{2}}{c_{3}}
$$

defined by $j\left(\tau+\tau_{2}\right)=2 j(\tau)$.

\section{E. Half-early-peak law}

The early differential rate exhibits a very interesting feature referred to here as the half-early-peak law. According to Eq. (23), the rate at half of the peak time $j_{1 / 2}=j\left(\tau_{m} / 2\right)$ is given by

$$
\frac{j_{1 / 2}}{j_{\max }}=\frac{4 c_{0} \cosh ^{2}\left(\frac{c_{3} \tau_{m}}{2}\right)}{\left(\sqrt{c_{0}}+\sqrt{j_{\max }}\right)^{2}}=\frac{4 j(0) \cosh ^{2}\left(\frac{c_{3} \tau_{m}}{2}\right)}{\left(\sqrt{j(0)}+\sqrt{j_{\max }}\right)^{2}}
$$

The corresponding cumulative half-early-peak law follows from the first case of Eq. (20) as

$$
J_{1 / 2}=J\left(\frac{\tau_{m}}{2}\right)=\eta+\frac{J_{0}-\eta}{1+\sqrt{\frac{j_{\max }}{c_{0}}}}
$$

or

$$
1+\sqrt{\frac{j_{\max }}{c_{0}}}=\frac{J_{0}-\eta}{J_{1 / 2}-\eta}
$$

With this equation the half-early-peak law (27) reads

$$
\begin{aligned}
\frac{j_{1 / 2}}{j_{\max }} & =\left[\frac{2\left(J_{1 / 2}-\eta\right) \cosh \left(\frac{c_{3} \tau_{m}}{2}\right)}{J_{0}-\eta}\right]^{2} \\
& =\left[\frac{2\left[J_{1 / 2}-J(0)\right] \cosh \left(\frac{c_{3} \tau_{m}}{2}\right)}{J_{0}-J(0)}\right]^{2}
\end{aligned}
$$

In case of temporal wave distributions with an apparent peak the half-early-peak law (30) provides an important test for the derived parameters of the wave as it relates directly the monitored quantities $J(0)=J(\tau=0), J_{0}=J\left(\tau_{m}\right), j_{1 / 2}, j_{\max }$ and $c_{3} \tau_{m} / 2$, where the latter can also be written in terms of the ratio between peak time $\tau_{m}$ and early doubling time $\tau_{2}$ as $c_{3} \tau_{m} / 2=\ln \left(2^{1 / 4}\right) \tau_{m} / \tau_{2} \approx \frac{1}{6} \tau_{m} / \tau_{2}$.

\section{F. Reintroducing dimensions: Real time evolution}

The rates (23) and the cumulative number (20) refer to the relative time $\tau$ defined in Eq. (1), whereas the monitored data refer to real time $t$. We therefore adopt, well justified for the initial phase of any new emergent wave, a constant infection rate $a(t)=a_{0}$ throughout so that $\tau=a_{0}\left(t-t_{0}\right)$, where for ease of exposition we drop the index $n$ and simply write $t_{0}=t_{0, n}$. We then obtain in real time for the peak time

$$
t_{m}=t_{0}+\frac{\tau_{m}}{a_{0}}
$$

Likewise, the pandemic time evolutions in real time follow readily from

$$
\begin{aligned}
& J(t)=J\left(\tau=a_{0}\left(t-t_{0}\right)\right), \\
& \dot{J}(t)=a_{0} j\left(\tau=a_{0}\left(t-t_{0}\right)\right),
\end{aligned}
$$

and are written down in Appendix B. Likewise, the doubling time at early times $t \ll t_{m}$ and the decay half-time at late times $t \gg t_{m}$ are given by

$$
\begin{aligned}
t_{1 / 2} & =\frac{\tau_{1 / 2}}{a_{0}} \approx \frac{0.693}{a_{0}\left[J_{\infty}-(1-k)\right]}, \\
t_{2} & =\frac{\tau_{2}}{a_{0}}=\frac{\ln \sqrt{2}}{a_{0} c_{3}} \approx \frac{0.347}{a_{0} c_{3}}
\end{aligned}
$$

whereas the half-early-peak law in real time becomes

$$
\frac{\dot{J}_{1 / 2}}{\dot{J}_{\max }}=\left[\frac{2\left(J_{1 / 2}-J\left(t_{0}\right)\right) \cosh \left[\frac{c_{3} a_{0}\left(t_{m}-t_{0}\right)}{2}\right]}{J_{0}-J\left(t_{0}\right)}\right]^{2}
$$

This will be made more precise below, as we apply the semitime SIR model to two waves, each with its own onset.

\section{APPLICATIONS}

The above derivations resulted in explicit expressions for the dimensionless fraction $J$ of infected persons and the dimensionless rate of infections $j$, both in terms of reduced time $\tau$, the inverse reproduction number $k$ and the initial condition $J(0)=\eta$. Measured, reliable data is usually available for the total number of deceased persons $D(t)$ as function of time $t$ in units of days, and the total population $N$ can be considered known. Since the number of deaths follows the number of infections with a delay of about 10 days, ${ }^{2}$ we can use $D(t)$ to make predictions about the number of infected persons at $t-10$ days.

To uniquely determine the model parameters from the data, which allows us then to draw conclusions about the future time-evolution of measurable quantities, we need to assume a fixed relationship between real and reduced time. To this end we adopt for the time during each of the pandemic waves a constant infection rate $a_{0}$ so that $\tau=\int_{t_{0}}^{t} d \xi a(\xi)=a_{0}\left(t-t_{0}\right)$ where $t_{0}$ is the real time marking the beginning of the $n$th wave, and $\tau$ the reduced time that vanishes at the beginning of the $n$th wave.

With the known fatality ratio of $f \approx 0.005$, the cumulative number of infected persons (including those, that have not been identified) is $f D(t) / N$. More precisely, $J(\tau)=$ $f D(t) / N$ during the first wave. Because the cumulative number accumulate during subsequent waves, it is more convenient to model the measured daily rate $\dot{J}(t)$ of newly infected persons instead of the cumulative numbers. More precisely, one has $j(\tau)=f \dot{D}(t) / a_{0} N$, and it is this dimensionless $j(\tau)$ which we have expressed in terms of $k$ and $\eta$ above, while $\eta$ is contained in the initial condition, $j(0)=\eta(1-\eta)=$ $f \dot{D}\left(t_{0}\right) / a_{0} N$. For each wave there are thus three parameters to be determined, $k, \eta$, and $a_{0}$, or alternatively, $k, t_{0}$, and $a_{0}$. 


\begin{tabular}{lllrrrrrrrrrrrr}
\hline \hline country & $\alpha_{3}$ & wave & $N / 10^{6}$ & $k$ & $\eta N$ & $a_{0}$ & $t_{0}$ & $t_{m}$ & $J_{\infty}$ & prec. & $J_{\infty} N$ & $t_{2}$ & $t_{1 / 2}$ & $D_{\infty}$ \\
\hline Italy & ITA & $\# 1$ & 60.6 & 0.942 & 294 & 1.49 & $2020-01-11$ & $2020-04-03$ & $11.4 \%$ & $7.3 \%$ & 6892608 & 8 & 8 & 34463 \\
Germany & DEU & $\# 1$ & 82.7 & 0.990 & 705 & 9.94 & $2020-03-16$ & $2020-04-16$ & $2.0 \%$ & $7.0 \%$ & 1644557 & 6 & 6 & 8223 \\
Switzerland & CHE & $\# 1$ & 8.4 & 0.978 & 283 & 5.12 & $2020-03-10$ & $2020-04-08$ & $4.6 \%$ & $8.5 \%$ & 385200 & 6 & 6 & 1926 \\
Great Britain & GBR & $\# 1$ & 65.6 & 0.939 & 306 & 1.52 & $2020-01-29$ & $2020-04-17$ & $12.0 \%$ & $7.0 \%$ & 7901797 & 7 & 8 & 39509 \\
France & FRA & $\# 1$ & 66.9 & 0.958 & 501 & 3.17 & $2020-02-23$ & $2020-04-09$ & $8.3 \%$ & $10.1 \%$ & 5546490 & 5 & 5 & 27732 \\
Belgium & BEL & $\# 1$ & 11.3 & 0.917 & 897 & 1.62 & $2020-03-07$ & $2020-04-13$ & $16.2 \%$ & $9.4 \%$ & 1840827 & 5 & 5 & 9204 \\
Canada & CAN & $\# 1$ & 36.3 & 0.976 & 579 & 2.90 & $2020-03-06$ & $2020-05-05$ & $4.9 \%$ & $6.1 \%$ & 1774863 & 5 & 10 & 8874 \\
Russia & RUS & $\# 1$ & 144.3 & 0.987 & 891 & 2.69 & $2020-03-05$ & $2020-06-25$ & $2.6 \%$ & $9.3 \%$ & 3753373 & 19 & 20 & 18767 \\
\hline Italy & ITA & $\# 2$ & 60.6 & 0.915 & 1095 & 0.71 & $2020-08-16$ & $2020-12-04$ & $27.8 \%$ & $6.5 \%$ & 16870360 & 12 & 13 & 84352 \\
Germany & DEU & $\# 2$ & 82.7 & 0.932 & 2129 & 0.75 & $2020-09-11$ & $2021-01-02$ & $15.4 \%$ & $7.8 \%$ & 12712430 & 15 & 16 & 63562 \\
Switzerland & CHE & $\# 2$ & 8.4 & 0.906 & 121 & 0.61 & $2020-08-03$ & $2020-12-04$ & $22.7 \%$ & $5.7 \%$ & 1904648 & 7 & 13 & 9523 \\
Great Britain & GBR & $\# 2$ & 65.6 & 0.839 & 1325 & 0.21 & $2020-06-04$ & $2021-01-15$ & $42.5 \%$ & $15.0 \%$ & 27884238 & 20 & 23 & 139421 \\
France & FRA & $\# 2$ & 66.9 & 0.932 & 4693 & 0.68 & $2020-08-16$ & $2020-11-27$ & $21.7 \%$ & $6.9 \%$ & 14532325 & 15 & 15 & 72662 \\
Belgium & BEL & $\# 2$ & 11.3 & 0.904 & 2360 & 0.70 & $2020-09-12$ & $2020-11-16$ & $34.9 \%$ & $5.6 \%$ & 3965235 & 10 & 11 & 19826 \\
Canada & CAN & $\# 2$ & 36.3 & 0.954 & 2345 & 0.69 & $2020-09-07$ & $2021-01-13$ & $14.1 \%$ & $5.1 \%$ & 5131542 & 21 & 22 & 25658 \\
Russia & RUS & $\# 2$ & 144.3 & 0.951 & 20158 & 0.58 & $2020-08-23$ & $2020-12-20$ & $12.4 \%$ & $5.7 \%$ & 17892267 & 23 & 24 & 89461 \\
\hline \hline
\end{tabular}

TABLE I. Results from the analysis of $n$th (first and second) pandemic waves in selected countries based on data from 11 Jan 2021 . The columns contain the population $N$, SIR parameters $k, \eta$, infection rate $a_{0}$, time of outbreak $t_{0}$, the resulting peak time $t_{m}$, fraction $J_{\infty}$ of cumulated infected persons at the end of the $n$th wave, the precision (prec.) to which the data was described by the semi-time SIR model, the total number $J_{\infty} N$ of infected persons at the end of the $n$th wave, the early doubling $\tau_{2}$ and late half decay times $\tau_{1 / 2}$ given by Eqs. (26) and (25), and the total number of projected fatalities $D_{\infty}$ at the end of the $n$th wave. All the polynomial coefficients $c_{0}, c_{1}, c_{2}, d_{1}, d_{2}$, and $j_{\text {max }}$, characterizing the relationship between daily new fraction, $j$, and cumulative fraction of infected persons, $J$, are expressed in terms of $\eta$ and $k$ within this manuscript.
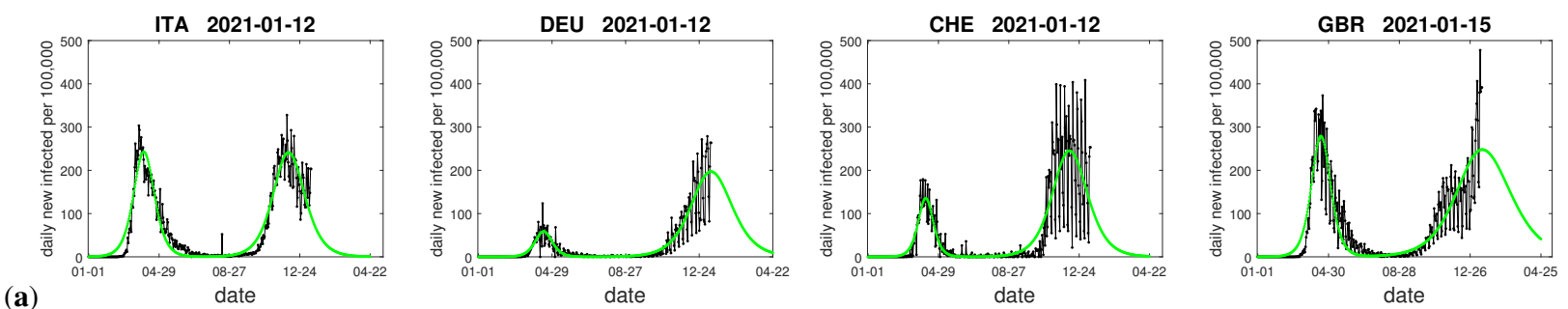

(a)
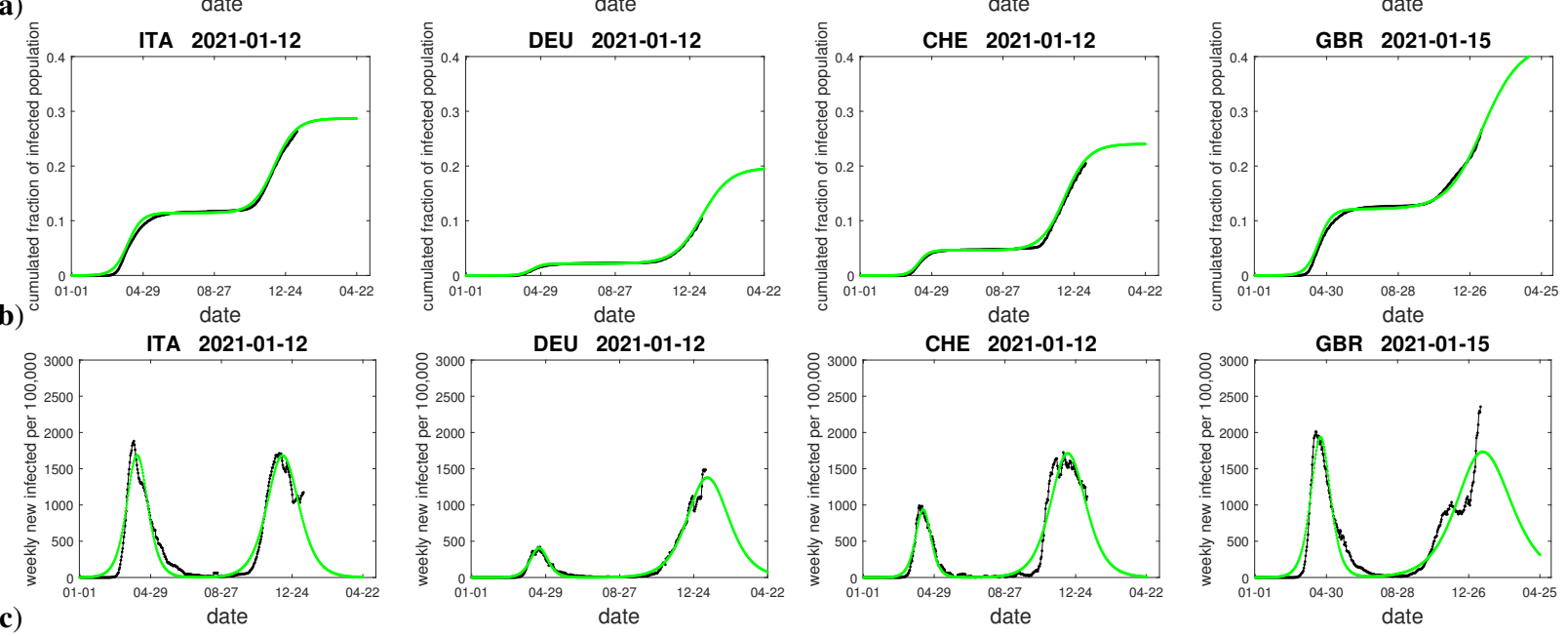

FIG. 1. Amount of infected persons, estimated from the reported fatality data for four countries (black), assuming a fatality rate of $f=0.005$, along with predictions resulting from the semi-time SIR model (green). The SIR parameters and related quantities are listed in Tab. I. (a) Daily new infected persons per 100,000 inhabitants (black), together with the SIR prediction (green). (b) Cumulated fraction of infected persons, and (c) weekly new infected persons per 100,000 inhabitants. Shown here is the analysis of data for Italy, Germany, Switzerland, and Great Britain. For additional countries see Fig. 2. 


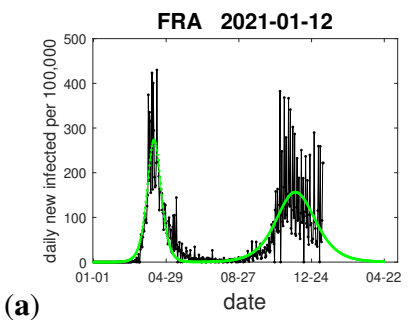

(a)

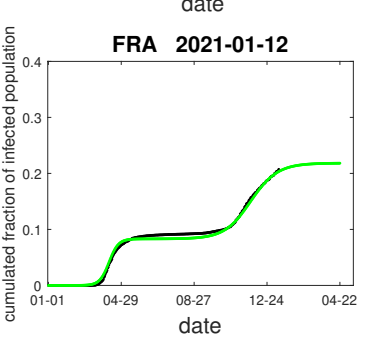

(b)

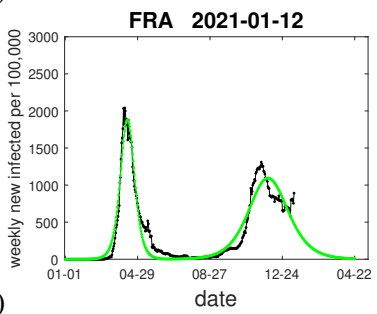

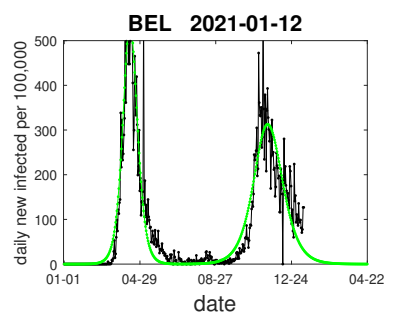
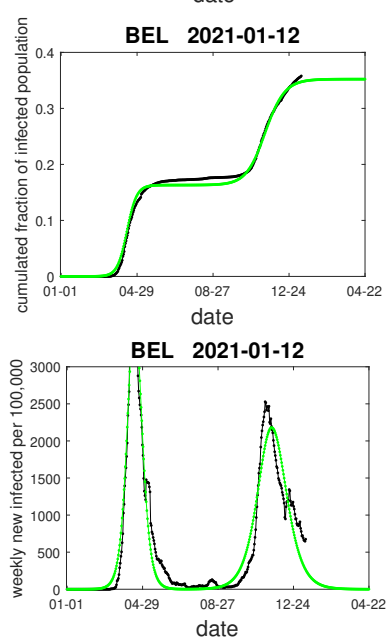

CAN 2021-01-12
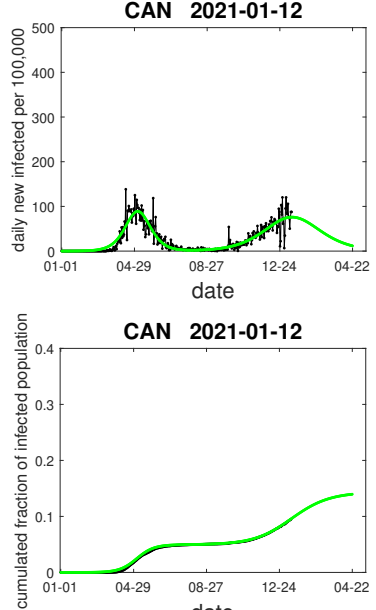

CAN 2021-01-12

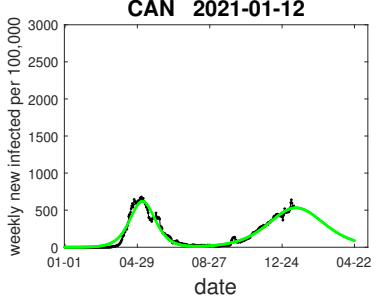

RUS 2021-01-12
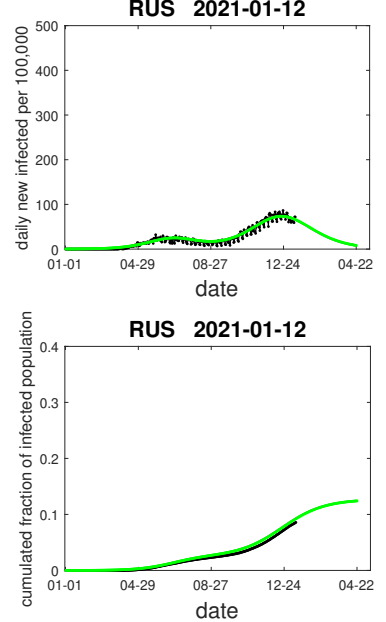

RUS 2021-01-12

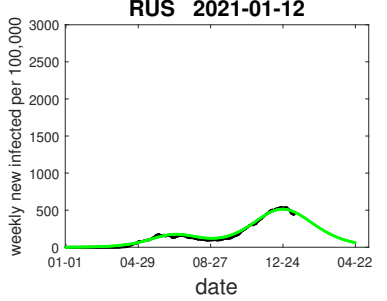

FIG. 2. Same as Fig. 1 for additional countries: France, Belgium, Canada, and Russia.

In practise, as the measured data is fluctuating considerably, especially at the beginning of a pandemic wave, it turns out to be even more convenient to work with four unknowns, $k, t_{0}, \eta$, and $a_{0}$, and to determine these parameters upon requiring that the absolute deviation between measured and predicted $j(\tau)$ achieves a global mininum for each wave separately. Start values for the global minimization are readily available from our above consideration about the early time evolution, the position of the maximum in real time, $t_{m}=t_{0}+a_{0} \tau_{m}$, the value of the dimensionless $J\left(\tau_{m}\right)=J_{0}=f D\left(t_{0}+\tau_{m} / a_{0}\right) / N$, where $\tau_{m}$ and $J_{0}$ are known in terms of $k$ and $\eta$. With the fitted parameters at hand the model-predicted cumulative fraction of infected persons in real time $t$ is $J(t)=\int_{t_{0}}^{t} \dot{J}(\xi) d \xi=$ $a_{0} \int_{t_{0}}^{t} j\left[a_{0}\left(t-t_{0}\right)\right] d t=\int_{0}^{\tau} j\left(\tau^{\prime}\right) d \tau^{\prime}$ and the model-predicted number of deceased persons is thus $D(t)=J(t) f N$.

For the sake of clarity, because the number of infections is $f^{-1}$ times the number of fatalities, and because the number of fatalities is more reliably reported than the true number of infections, we do not add any delay time when presenting figures, so that the time of outbreak of the pandemic can be considered 10 days earlier than $t_{0}$. Similarly, the peak time of daily new infections is roughly 10 days earlier than the tabulated $t_{m}$, which is the peak time of daily fatalities.

In the following we determine the two sets of four SIR parameters by fitting the early time evolution before the peaks of first and second waves with the monitored early daily case rates $j(t)$ during the waves. The obtained numbers are tabulated in Tab. I for selected countries, while the procedure has been applied to more than 100 countries during the course of this study (Supplementary website). The measured data is compared with the SIR predictions in Fig. 1. With the four coefficients $c_{1}, c_{2}, t_{0}$ and $a_{0}$ determined we then can infer

(1) the values of the parameters $\eta$ and $k$ characterizing the waves,

(2) the final number of infected persons $N J_{\infty}$, the maximum daily rate $j_{\max }$ at the peak time $\tau_{m}$ according to Eqs. (4), (7), and (18), and

(3) the late time evolution of the second wave after its peak from Eqs. (20) and (32) determined by the coefficients $d_{1}$ and $d_{2}$ given uniquely by Eqs. (12) and (15) in terms of $k, J_{\infty}, c_{0}$, $c_{1}$ and $c_{2}$, which in turn are given by Eqs. (10), (11), (14), i.e. finally, in terms of $k$, the initial condition $\varepsilon$, and reduced time $\tau$. All these results are collected in Tab. I.

Despite the differences in the response of authorities to the emerging pandemic, length and completeness of lock-downs etc. there are very comparable patterns for all countries. Both the inverse reproduction number $k$ and the infection rates $a_{0}$ have significantly dropped during the 2 nd wave as compared with the first wave. The decreasing $k=\mu_{0} / a_{0}$ tends to increase the peak height, while the decreasing $a_{0}$ tends to lower it. The reduction of $a_{0}$ comes as a surprise to us, but a decrease of $a_{0}$ is in agreement with the observed broadening of the 2nd wave.

One likely explanation maybe that the early phases of the 2nd waves in all considered countries occurred under light lockdown conditions whereas no lockdown measures have been taken during the initial phases of the first wave. Moreover, the infection rates $a_{0}$ of the second wave have dropped as a result of the overall, increasingly cautious, self-protective behavior of the population.

While the 2nd peak times are comparable in Italy, Switzer- 
land, France, and Russia, they are delayed in Canada, Germany, Great Britain. On the positive side, according to our analysis, the peak time $t_{m}$ of the 2nd wave has passed already in France, Belgium, Italy, Germany, Switzerland, and Russia, and more than half of the population in Great Britain will have been infected already after the 2nd wave, thus getting closer to herd immunity. In general, the 2nd wave increased the immunity more significantly than the first wave, as is obvious from the sharp rise of $J_{\infty}$ between 1st and 2nd wave (Tab. I). The last column in this table contains the SIR predictions for the number of fatalities at the end of the 2 nd wave. While countries like Germany was hit by a moderately low number of fatalities during the first wave, it will be increasing by a factor of about 7 during the 2 nd wave, despite rigorous interventions. Still, the fraction of seriously affected population remains slightly below in this country compared with countries like Switzerland, that did not install a similarly rigorous scenario with hard lock-downs. The measured data is captured by the semi-time SIR model by a relative error of about 5-10\%. At present. the situation in Great Britain is obviously the darkest for these sets of data. The trend is most likely caused by the recent, more transmittable variant (SARS-CoV2 VUI 202012/01) of the virus. As of today (Jan. 13, 2021) the peak time in the death rate in Great Britain is still ahead.

Within the semi-time SIR model, the exponential behavior of the differential rate of newly infected persons $j(\tau)$ is characterized by the early doubling time $\tau_{2}$ and the late half decay times $\tau_{1 / 2}$, which we have included in Tab. I. Their analytic expressions were given by Eqs. (26) and (25). It seems that $\tau_{1 / 2}$ is for all cases reported in our table approximately equal to the doubling time $\tau_{2}$. To see, if this is a generic feature of the SIR model, one has to just inspect the ratio $\tau_{2} / \tau_{1 / 2}=\left(J_{\infty}-1+k\right) / 2 c_{3}$, using the above explicit expressions for $j_{\max }$ and $c_{3}$. For $k$ close to unity, and small $\eta \ll 1$, the ratio evaluates to

$$
\lim _{\eta \rightarrow 0} \frac{\tau_{2}}{\tau_{1 / 2}}=\frac{1+2 k}{3}
$$

in agreement with Tab. (I), as $k$ is sufficiently close to unity. To stress an important aspect, and because we can take the opportunity to compare with related recent work,${ }^{34}$ the ratio over the whole $k$ range is shown in Fig. 3 for various finite $\eta$. This ratio reflects the asymmetry of the pandemic wave, as $J_{0}-J_{\infty} / 2$ does. It is important to notice here that the initial condition affects not only the asymmetry of the wave, but the asymptotic behavior qualitatively. The corresponding result for the all-time SIR model is shown for comparison. It is however recovered by the semi-time SIR for sufficiently small $\eta$. The appearance and relevance of power law and Gaussian rather than exponential tails, that are not predicted by the SIR model with time-independent $k$, had been discussed in several recent works. ${ }^{1-4,37}$ Early-outbreak estimates of $k$ and their uncertainty have been discussed in detail. ${ }^{38}$

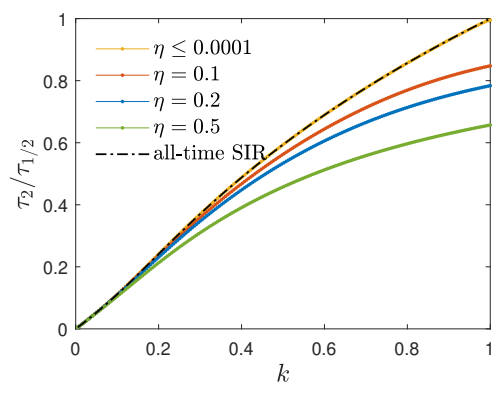

FIG. 3. Ratio between early doubling time $\tau_{2}$ and late half decay time $\tau_{1 / 2}$ versus $k$, for various initial conditions. For comparison, we include the corresponding result ${ }^{34}$ for the all-time SIR model, $\tau_{2} / \tau_{1 / 2}=k\left[1+W_{0}\left(-k^{-1} e^{-1 / k}\right)\right] /(1-k)$.

\section{SUMMARY AND CONCLUSIONS}

We have derived simple analytic expressions for all measurable amounts of cases and fatalities during a pandemic evolution described by the semi-time SIR model, that share all relevant features with the exact solution of the semi-time SIR model, including time and position of the peak of daily new infections, as well as the asymptotic behaviors at small and large times. We show, in particular, how the asymmetry of the epidemic wave and its exponential tails are affected by the initial conditions; a feature that has no analogue in the all-time SIR model. The expressions are so precise that they can be used instead of a numerical solution of the SIR model. The advantage of an analytical expression is obvious, as it allows to quickly determine the SIR parameters from the measured data well ahead the peak time, and thus allows for predictions that serve as a prerequisite to make decisions. We applied the approach to eight different countries from different continents. We summarized the exact features of the semi-time SIR model, stated the approximants for the reliably measurable quantities, and collected all the derivations of the new approximant in an appendix. Our analysis reveals that the immunity is very strongly increasing during the 2 nd wave, while it was still at a very moderate level of a few percent in several countries at the end of the first wave. The wave-specific SIR parameters $\mu_{0}$ and $a_{0}$ describing the infection and recovery rates we find to behave in a similar fashion, while their ratio $k=\mu_{0} / a_{0}$ was decreasing only by a about $5 \%$ for the countries mentioned in Tab. I and Figs. 1 and 2. Still, an apparently moderate change of $k$ can have significant consequences for the relevant numbers like the final amount of infected or deceased population, captured by $J_{\infty}$. For $k$ close to unity and small $\eta \ll 1$, our results imply $j_{\max } \approx \frac{1}{2}(1-k)^{2}$ and $J_{\infty} \approx 2(1-k)$, c.f., Fig. 4 . This implies a typical duration of the differential fraction of newly infected persons, $w \approx J_{\infty} / a_{0} j_{\max } \approx 4 / a_{0}(1-k)$, that decreases dramatically with decreasing $k$, but increases with decreasing $a_{0}$. It is this qualitative feature of the SIR model, that leads to its counterintuitive parameters we reveal having analyzing the two pandemic waves, and that has to be taken into account when speculating about possible additional waves. As we have shown, 
the probability for an additional wave is however low in several countries due to the fraction of immune inhabitants at the end of the 2nd wave, irrespective the currently ongoing vaccination efforts.

\section{Acknowledgment}

M.K. thanks the Swiss National Supercomputing Centre for providing computational resources (CSCS projects go11 and s987).

\section{Data availability statement}

The data used to create all graphs that mentioned reported numbers were collected from the following website at github: https://pomber.github.io/covid19/timeseries.json

\section{Competing interests}

There are no competing interests to declare.

\section{Funding}

This research did not receive any particular funding.

\section{Author's contributions}

Both authors contributed equally to this work.

\section{Supplementary material}

The analysis has been performed for many additional countries. Results are available online at https://www.complexfluids.ethz.ch/covid19-wave2.

\section{Appendix A: Calculation of integrals}

Eq. (19) can be written as

$$
\tau-\tau_{m} \simeq \begin{cases}-I_{c} & \text { for } \tau \leq \tau_{m} \\ I_{d} & \text { for } \tau \geq \tau_{m}\end{cases}
$$

in terms of the three integrals $\tau_{m}, I_{c}$, and $I_{d}$,

$$
\begin{aligned}
\tau_{m} & =\int_{\eta}^{J_{0}} \frac{d y}{j_{c}(y)}=\int_{\eta}^{J_{0}} \frac{d y}{c_{0}+c_{1}(y-\eta)+c_{2}(y-\eta)^{2}} \\
& =\int_{0}^{J_{0}-\eta} \frac{d x}{c_{0}+c_{1} x+c_{2} x^{2}}, \\
I_{c} & =\int_{J}^{J_{0}} \frac{d y}{j_{c}(y)}=\int_{J-\eta}^{J_{0}-\eta} \frac{d x}{c_{0}+c_{1} x+c_{2} x^{2}} \\
I_{d} & =\int_{J_{0}}^{J} \frac{d y}{j_{d}(y)}=\int_{J}^{J_{0}} \frac{d y}{d_{1}\left(J_{\infty}-y\right)+d_{2}\left(J_{\infty}-y\right)^{2}} \\
& =\int_{J_{\infty}-J}^{J_{\infty}-J_{0}} \frac{d x}{x\left(d_{1}+d_{2} x\right)}=\int_{\frac{1}{J_{\infty}-J}}^{\frac{1}{J_{\infty}-J_{0}}} \frac{d w}{d_{2}+d_{1} w},(\mathrm{~A})
\end{aligned}
$$

where in the last step we substituted $x=1 / w$. The integral (A4) then becomes

$$
I_{d}=\frac{1}{d_{1}} \ln \frac{1+\frac{d_{1}}{d_{2}\left(J_{\infty}-J\right)}}{1+\frac{d_{1}}{d_{2}\left(J_{\infty}-J_{0}\right)}}
$$

As the coefficient $c_{2}<0$ is negative the expression

$$
2 c_{3}=\sqrt{c_{1}^{2}-4 c_{0} c_{2}}>0
$$

is always real-valued and positive. Consequently, the two integrals (A2) and (A3) are given by

$$
\begin{aligned}
\tau_{m} & =\frac{1}{2 c_{3}}\left[\ln \frac{c_{2} x+\frac{c_{1}-2 c_{3}}{2}}{c_{2} x+\frac{c_{1}+2 c_{3}}{2}}\right]_{0}^{J_{0}-\eta} \\
& =\frac{1}{2 c_{3}}\left[\ln \frac{1-\frac{2 c_{3}}{c_{1}+c_{2} x}}{1+\frac{2 c_{3}}{c_{1}+2 c_{2} x}}\right]_{0}^{J_{0}-\eta} \\
& =-\frac{1}{c_{3}}\left[\operatorname{artanh} \frac{2 c_{3}}{c_{1}+2 c_{2} x}\right]_{0}^{J_{0}-\eta} \\
& =\frac{1}{c_{3}}\left[\operatorname{artanh} \frac{2 c_{3}}{c_{1}}-\operatorname{artanh} \frac{2 c_{3}}{c_{1}+2 c_{2}\left(J_{0}-\eta\right)}\right] \\
& =\frac{1}{c_{3}}\left[\operatorname{artanh} \frac{4 c_{2} c_{3}\left(J_{0}-\eta\right)}{c_{1}^{2}-4 c_{3}^{2}+2 c_{1} c_{2}\left(J_{0}-\eta\right)}\right] \\
& =\frac{1}{c_{3}} \operatorname{artanh} \frac{2 c_{3}}{c_{1}+\frac{2 c_{0}}{J_{0}-\eta}}
\end{aligned}
$$

and

$$
\begin{aligned}
I_{c} & =\frac{1}{2 c_{3}}\left[\ln \frac{c_{2} x+\frac{c_{1}-2 c_{3}}{2}}{c_{2} x+\frac{c_{1}+2 c_{3}}{2}}\right]_{J-\eta}^{J_{0}-\eta} \\
& =\frac{1}{c_{3}}\left[\operatorname{artanh} \frac{2 c_{3}}{c_{1}+2 c_{2} x}\right]_{J_{0}-\eta}^{J-\eta} \\
& =\frac{1}{c_{3}} \operatorname{artanh} \frac{4 c_{2} c_{3}\left(J_{0}-J\right)}{c_{1}^{2}-4 c_{3}^{2}+2 c_{1} c_{2} \beta_{1}+4 c_{2}^{2} \beta_{2}} \\
& =\frac{1}{c_{3}} \operatorname{artanh} \frac{2 c_{3}\left(J_{0}-J\right)}{2 c_{0}+c_{1} \beta_{1}+2 c_{2} \beta_{2}},
\end{aligned}
$$

where in both calculations we made use of Eq. (A6) in the last step. To make the expression (A8) more readable, we used the 

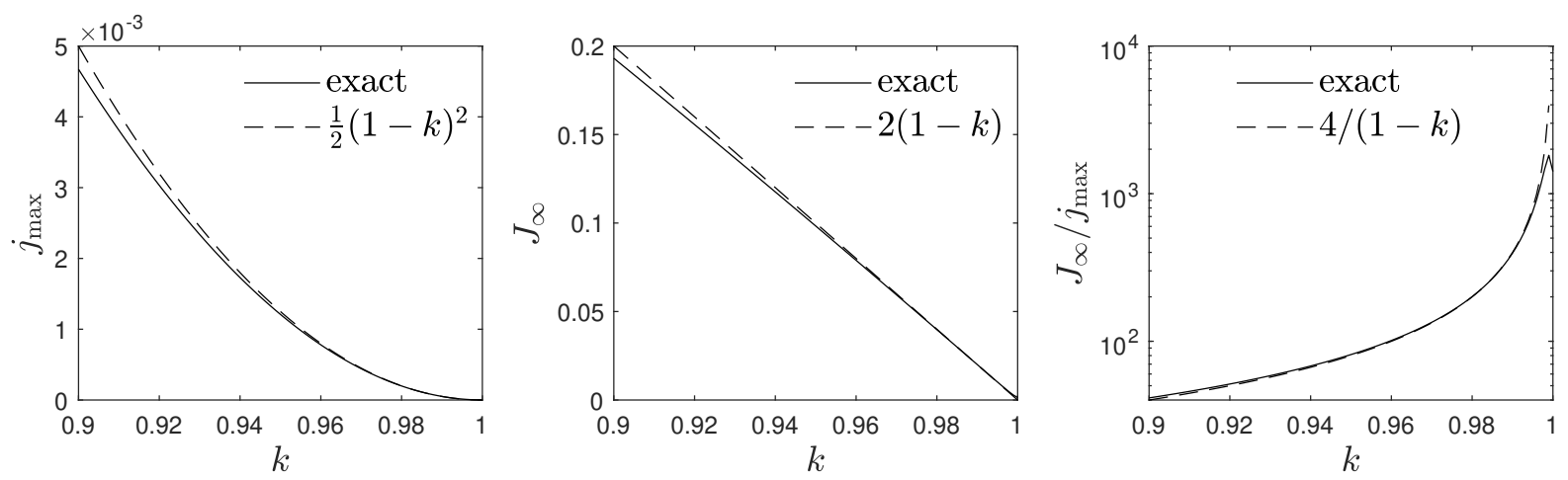

FIG. 4. (Exact behavior (solid lines) of the peak differential fraction of infected persons $j_{\max }$, the final fraction of infected persons $J_{\infty}$, and the ratio $J_{\infty} / j_{\max }$, that characterizes the dimensionless width of the wave. All curves are shown to be well captured by the analytic expressions mentioned in the legends (dashed lines), that we use to discuss qualitatively, in concert with the dimensional infection rate $a_{0}$, the obtained SIR parameters. The exact expressions for $j_{\max }$ and $J_{\infty}$ are given in terms of Lambert's function in Eqs. (7) and (4). The plot is done with $\eta=10^{-6}$, but it is essentially unaffected by the choice of $\eta$ as long as $\eta \ll 1$.

abbreviations $\beta_{1}=J_{0}+J-2 \eta$ and $\beta_{2}=\left(J_{0}-\eta\right)(J-\eta)$. As an aside, we note that for $J=\eta$ the last formula (A8) correctly reduces to Eq. (A7).

\section{Early reduced times $\tau \leq \tau_{m}$}

Collecting terms in Eq. (A1) we obtain for early reduced times $\tau \leq \tau_{m}$ with the abbreviation $X=c_{3}\left(\tau_{m}-\tau\right)$ as well as

$$
\begin{aligned}
Y & =J-\eta, \quad Y_{0}=J_{0}-\eta, \\
T(\tau) & =-\frac{1}{2 c_{3}} \tanh (X)
\end{aligned}
$$

the linear relation

$$
\begin{aligned}
Y-Y_{0} & =\left[2 c_{0}+c_{1}\left(Y+Y_{0}\right)+2 c_{2} Y Y_{0}\right] T \\
& =\left[2 c_{0}+c_{1} Y_{0}+\left(c_{1}+2 c_{2} Y_{0}\right) Y\right] T
\end{aligned}
$$

Inserting Eq. (14) for $c_{2}$ we find

$$
c_{1}+2 c_{2} Y_{0}=\frac{2 j_{\max }-\left(2 c_{0}+c_{1} Y_{0}\right)}{Y_{0}},
$$

simplifying Eq. (A11) to

$$
Y-Y_{0}=\left[\left(2 c_{0}+c_{1} Y_{0}\right)\left(1-\frac{Y}{Y_{0}}\right)+\frac{2 j_{\max } Y}{Y_{0}}\right] T
$$

From Eqs. (A10) and (A7) we identify

$$
T(0)=T(\tau=0)=-\frac{Y_{0}}{2 c_{0}+c_{1} Y_{0}},
$$

so that Eq. (A13) becomes

$$
\frac{2 j_{\max } Y}{Y_{0}\left(Y-Y_{0}\right)}=\frac{1}{T}-\frac{1}{T(0)}
$$

Eq. (A15) is solved by

$$
Y(T)=\frac{Y_{0}}{1-\frac{A}{\left(\frac{1}{T}-\frac{1}{T(0)}\right)}}=\frac{Y_{0}}{1+\frac{A T(0) T}{T-T(0)}},
$$

with

$$
A=\frac{2 j_{\max }}{Y_{0}}
$$

Using Eq. (A10) then provides

$$
\begin{aligned}
\frac{1}{T}-\frac{1}{T(0)} & =2 c_{3}\left[\operatorname{coth}(X)+\operatorname{coth}\left(c_{3} \tau_{m}\right)\right] \\
& =-2 c_{3} \frac{\sinh \left(c_{3} \tau\right)}{\sinh (X) \sinh \left(c_{3} \tau_{m}\right)}
\end{aligned}
$$

Consequently, Eq. (A16) reduces to

$$
\begin{aligned}
Y\left(\tau \leq \tau_{m}\right) & =\frac{Y_{0}}{1+\frac{A}{2 c_{3}} \frac{\sinh (X) \sinh \left(c_{3} \tau_{m}\right)}{\sinh \left(c_{3} \tau\right)}} \\
& =\frac{Y_{0}}{1+\frac{j_{\max }}{Y_{0} c_{3}} \frac{\sinh (X) \sinh \left(c_{3} \tau_{m}\right)}{\sinh \left(c_{3} \tau\right)}}
\end{aligned}
$$

Using $\tau_{m}$ from Eq. (A7) we find

$$
\begin{aligned}
\sinh \left(c_{3} \tau_{m}\right) & =\frac{\tanh \left(c_{3} \tau_{m}\right)}{\sqrt{1-\tanh ^{2}\left(c_{3} \tau_{m}\right)}} \\
& =\frac{c_{3} Y_{0}}{\sqrt{c_{0}^{2}+c_{0} c_{1} Y_{0}+c_{0} c_{2} Y_{0}^{2}}} \\
& =\frac{c_{3} Y_{0}}{\sqrt{c_{0}\left[c_{0}+c_{1} Y_{0}+c_{2} Y_{0}^{2}\right]}}
\end{aligned}
$$

According to the first Eq. (16)

$$
j_{\max }=j\left(J_{0}\right)=c_{0}+c_{1} Y_{0}+c_{2} Y_{0}^{2}
$$

so that Eq. (A20) becomes

$$
\sinh \left(c_{3} \tau_{m}\right)=\frac{c_{3} Y_{0}}{\sqrt{c_{0} j_{\max }}}
$$

implying for Eq. (A19)

$$
Y\left(\tau \leq \tau_{m}\right)=\frac{Y_{0}}{1+\sqrt{\frac{j_{\max }}{c_{0}}} \frac{\sinh (X)}{\sinh \left(c_{3} \tau\right)}}
$$


and hence

$$
J\left(\tau \leq \tau_{m}\right)=\eta+\frac{J_{0}-\eta}{1+\sqrt{\frac{j_{\max }}{c_{0}}} \frac{\sinh (X)}{\sinh \left(c_{3} \tau\right)}}
$$

confirming the 1st case in Eq. (20). For $\tau=\tau_{m}$ this solution correctly reduces to $J_{0}$, whereas for $\tau=0$ it correctly reduces to $\eta$. Taking the derivative of Eq. (A24) we obtain the corresponding rate

$$
\begin{aligned}
j\left(\tau \leq \tau_{m}\right)= & \frac{d J\left(\tau \leq \tau_{m}\right)}{d \tau}=\frac{Y_{0} c_{3} \sqrt{\frac{j_{\max }}{c_{0}}}}{\left[1+\sqrt{\frac{j_{\max }}{c_{0}}} \frac{\sinh (X)}{\sinh \left(c_{3} \tau\right)}\right]^{2}} \\
& \times \frac{\sinh \left(c_{3} \tau\right) \cosh (X)+\cosh \left(c_{3} \tau\right) \sinh (X)}{\sinh ^{2}\left(c_{3} \tau\right)} \\
= & \frac{Y_{0} c_{3} \sqrt{\frac{j_{\max }}{c_{0}}}}{\left[1+\sqrt{\frac{j_{\max }}{c_{0}}} \frac{\sinh (X)}{\sinh \left(c_{3} \tau\right)}\right]^{2}} \frac{\sinh \left(c_{3} \tau_{m}\right)}{\sinh ^{2}\left(c_{3} \tau\right)} \\
= & j_{\max }\left(\frac{\sinh \left(c_{3} \tau_{m}\right)}{\sinh \left(c_{3} \tau\right)+\sqrt{\frac{j_{\max }}{c_{0}}} \sinh (X)}\right)^{2}
\end{aligned}
$$

where we used Eq. (A22) and the earlier introduced abbreviation $X=c_{3}\left(\tau_{m}-\tau\right)$. The same result is obtained if we insert $J(\tau)$ from Eq. (20) into (16). This expression (A25) confirms the first case in Eq. (23).

\section{Late reduced times $\tau \geq \tau_{m}$}

Likewise, for large reduced times $\tau \geq \tau_{m}$ we obtain for Eq. (2)

$$
\left[1+\frac{d_{1}}{d_{2}\left(J_{\infty}-J_{0}\right)}\right] e^{d_{1}\left(\tau-\tau_{m}\right)}-1=\frac{d_{1}}{d_{2}\left(J_{\infty}-J\right)},
$$

yielding

$$
J\left(\tau \geq \tau_{m}\right)=J_{\infty}-\frac{J_{\infty}-J_{0}}{\left(1+\frac{d_{2}\left(J_{\infty}-J_{0}\right)}{d_{1}}\right) e^{d_{1}\left(\tau-\tau_{m}\right)}-\frac{d_{2}\left(J_{\infty}-J_{0}\right)}{d_{1}}}
$$

From Eq. (15) we find

$$
\frac{d_{2}\left(J_{\infty}-J_{0}\right)}{d_{1}}=\frac{j_{\max }}{d_{1}\left(J_{\infty}-J_{0}\right)}-1
$$

so that

$$
J\left(\tau \geq \tau_{m}\right)=J_{\infty}-\frac{J_{\infty}-J_{0}}{\frac{j_{\max }}{d_{1}\left(J_{\infty}-J_{0}\right)}\left[e^{d_{1}\left(\tau-\tau_{m}\right)}-1\right]+1}
$$

in agreement with the 2 nd case in Eq. (20). The late time solution correctly provides $J_{0}$ for $\tau=\tau_{m}$ and $J_{\infty}$ in the limit $\tau \rightarrow \infty$.

Taking the derivative of Eq. (A29) with respect to $\tau$, the corresponding rate becomes

confirming the 2nd case in Eq. (23).

\section{Appendix B: Dynamics in real time}

As data is usually available in real time $t$, we here write down the cumulative and differential rates of newly infected persons, which follow from their dimensionless counterparts Eqs. 20 and (23), upon using the transformation (32). The cumulative number of infected persons at time $t$ is

$$
J(t) \simeq \begin{cases}\eta+\frac{J_{0}-\eta}{1+\sqrt{\frac{j_{\max }}{c_{0}} \frac{\sinh \left[c_{3} a_{0}\left(t_{m}-t\right)\right.}{\sinh \left(c_{3} a_{0}\left(t-t_{0}\right)\right)}}} & \text { for } t \leq t_{m} \\ J_{\infty}-\frac{J_{\max }}{d_{1}\left(J_{\infty}-J_{0}\right)}\left[e^{d_{1} a_{0}\left(t-t_{m}\right)}-1\right]+1 & \text { for } t \geq t_{m}\end{cases}
$$

and the differential rate $\dot{J}(t)=d J(t) / d t$ reads

$$
\frac{\dot{J}(t)}{a_{0} j_{\max }}= \begin{cases}\left(\frac{\sinh \left[c_{3} a_{0}\left(t_{m}-t_{0}\right)\right]}{\sinh \left[c_{3} a_{0}\left(t-t_{0}\right)\right]+\sqrt{\frac{j_{\max }}{c_{0}}} \sinh \left[c_{3} a_{0}\left(t_{m}-t\right)\right]}\right)^{2} & \text { for } t \leq t_{m} \\ \frac{e^{d_{1} a_{0}\left(t-t_{m}\right)}}{\left(1+\frac{j_{\max }}{d_{1}\left(J_{\infty}-J_{0}\right)}\left[e^{d_{1} a_{0}\left(t-t_{m}\right)}-1\right]\right)^{2}} & \text { for } t \geq t_{m}\end{cases}
$$

where all coefficients $c_{0}, c_{3}, d_{1}, J_{0}, J_{\infty}, j_{\max }$ are given in terms of $k$ and $\eta=J(0)$ in the above Eqs. (10), (22), (12), (5), (4), and (7), respectively.

\section{Appendix C: Quality of the approximant}

Here we evaluate the quality of the analytic approximant for the solution $j(\tau)$ of the semi-time SIR model. These calculation can be done for all remaining SIR quantities $S(\tau)$, $I(\tau), R(\tau)$ as well as $J(\tau)$, but the results are very comparable. In Fig. 5a we show the reduced time evolution of $j(\tau)$ for various $k$. The exact solution (solid black) is visually matching the approximant (23) (solid red). For comparison we include (dashed red) the $j(\tau)$ calculated via the identity (3). The relative error of the approximant, defined by the difference between approximant and exact solution, divided by $j_{\max }$, is shown in Fig. $5 \mathrm{~b}$, for the same $k$ 's, and again for our approximant (solid) and for the $j(\tau)$ calculated from the approximant $J(\tau)$ given by Eq. (20), and inserted into Eq. (3). The relative error of the approximant is below $2 \%$ for the relevant regime of $k \geq 8$, and drops to about $5 \%$ for $k=0.6$. It is important to realize from Fig. 5b that the error is only large during a small interval in time, but afterwards vanishes again, so that the deviations are negligible for any practial purposes. Similarly, deviations between exact and analytic approximant for $J(\tau)$ vanish exactly at $\tau=\tau_{m}$ and for both 
small $\tau \ll \tau_{m}$, and large times $\tau \gg \tau_{m}$. This means for the differential rate $j(\tau)$, that deviations are not accumulating, but compensating in time. Since the measured data is also fluctuating by amounts that easily exceed $10 \%$ between subsequent days, the precision can be considered excellent. For the readers convenience we include a similar comparison for the so-called version II approximant we derived in our pre- vious work (Fig. 6). The present approximant has the neat features that it exactly reaches the exact $j_{\max }$ at peak time $\tau_{m}$, and that it possesses the correct asymptotic behavior, which is not fully reflected by watching the relative error. Furthermore, the present approximant has a smaller error than the so-called version I approximant. ${ }^{35}$, and a simpler analytic form than the so-called version II approximant. ${ }^{35}$
1. Schlickeiser R, Schlickeiser F. A Gaussian model for the time development of the Sars-Cov-2 corona pandemic disease. Predictions for Germany made on March 30. Physics. 2020;2:164170.

2. Schüttler J, Schlickeiser R, Schlickeiser F, Kröger M. Covid-19 predictions using a Gauss model, based on data from April 2. Physics. 2020;2:197-202.

3. Lixiang L, Yang Z, Dang Z, Meng C, Huang J, Meng H, et al. Propagation analysis and prediction of the Covid-19. Infect Disease Model. 2020;5:282-292.

4. Ciufolini I, Paolozzi A. Mathematical prediction of the time evolution of the Covid-19 pandemic in Italy by a Gauss error function and Monte Carlo simulations. Eur Phys J Plus. 2020;135:355.

5. Schlickeiser R, Kröger M. Dark numbers and herd immunity of the first Covid-19 wave and future social interventions. Epidem Int J. 2020;4:000152.

6. Kendall DG. Deterministic and stochastic epidemics in closed populations. In: Proc. Third Berkeley Symp. on Math. Statist. and Prob.. vol. 4. Univ. of Calif. Press, Berkeley, United States; 1956. p. 149-165.

7. Kermack WO, McKendrick AG. A contribution to the mathematical theory of epidemics. Proc Royal Soc A. 1927;115:700721.

8. Keeling MJ, Rohani P. Modeling Infectious Diseases in Humans and Animals. Princeton University Press, Princeton, USA; 2008.

9. E E. Covid-19 and Sars-Cov-2. Modeling the present, looking at the future. Phys Rep. 2020;869:1.

10. Schlickeiser R, Kröger M. Epidemics forecast from SIRmodeling, verification and calculated effects of lockdown and lifting of interventions. Front Phys. 2020.

11. Kumar S, Ahmadian A, Kumar R, Kumar D, Singh J, Baleanu D, et al. An efficient numerical method for fractional SIR epidemic model of infectious disease by using Bernstein wavelets. Math. 2020;8:558.

12. Postnikov EB. Estimation of COVID-19 dynamics "on a backof-envelope?: Does the simplest SIR model provide quantitative parameters and predictions? Chaos Solitons Fractals. 2020;135.

13. Kochanczyk M, Grabowski F, Lipniacki T. Dynamics of COVID-19 pandemic at constant and time-dependent contact rates. Math Model Nat Phenomena. 2020;15:28.

14. Khaleque A, Sen P. The susceptible-infected-recovered model on a Euclidean network. J Phys A. 2013;46:095007.

15. Tome T, de Oliveira MJ. Susceptible-infected-recovered and susceptible-exposed-infected models. J Phys A. 2011;44:095005.

16. Long Y, Wang L. Global dynamics of a delayed two-patch discrete SIR disease model. Comm Nonlinear Sci Numer Simul. 2020;83:105117.

17. Lahrouz A, Settati A, El Fatini M, Pettersson R, Taki R. Probability analysis of a perturbed epidemic system with relapse and cure. Int J Comput Meth. 2020;17.
18. Liu F, Li X, Zhu G. Using the contact network model and Metropolis-Hastings sampling to reconstruct the COVID-19 spread on the "Diamond Princess". Sci Bull. 2020;65:12971305.

19. Gollier C. Pandemic economics: optimal dynamic confinement under uncertainty and learning. Geneva Risk Insur Rev. 2020;45:80-93.

20. Kessler DA, Shnerb NM. Novel exponents control the quasideterministic limit of the extinction transition. J Phys A. 2008;41:292003.

21. Naik PA, Zu J, Ghoreishi M. Stability analysis and approximate solution of SIR epidemic model with Crowley-Martin type functional response and holling type-II treatment rate by using homotopy analysis method. J Appl Anal Comput. 2020;10:14821515.

22. Chatterjee S, Sarkar A, Chatterjee S, Karmakar M, Paul R. Studying the progress of COVID-19 outbreak in India using SIRD model. Indian J Phys. 2020.

23. Comunian A, Gaburro R, Giudici M. Inversion of a SIR-based model: A critical analysis about the application to COVID-19 epidemic. Physica D. 2020;413:132674.

24. Croccolo F, Roman HE. Spreading of infections on random graphs: A percolation-type model for COVID-19. Chaos Solitons Fractals. 2020;139:110077.

25. Boccara N, Cheong K. Automata network SIR models for the spread of infectious-diseases in populations of moving individuals. J Phys A. 1992;25:2447-2461.

26. Azam S, Macias-Diaz JE, Ahmed N, Khan I, Iqbal MS, Rafiq M, et al. Numerical modeling and theoretical analysis of a nonlinear advection-reaction epidemic system. Comp Meth Progr Biomed. 2020;193:105429.

27. Chekroun A, Kuniya T. An infection age-space structured SIR epidemic model with Neumann boundary condition. Applicable Anal. 2020;99:1972-1985.

28. Khan MA, Ismail M, Ullah S, Farhan M. Fractional order SIR model with generalized incidence rate. AIMS Math. 2020;5:1856-1880.

29. Zohdi T I. An agent-based computational framework for simulation of global pandemic and social response on planet X. Comput Mech. 2020;66:1195-1209.

30. Chen X, Li J, Xiao C, Yang P. Numerical solution and parameter estimation for uncertain SIR model with application to COVID19. Fuzzy Optim Decision Making. 2020.

31. Fokas AS, Dikaios N, Kastis GA. Mathematical models and deep learning for predicting the number of individuals reported to be infected with SARS-CoV-2. J R Soc Interface. 2020;17(169):20200494.

32. Barlow NS, Weinstein SJ. Accurate closed-form solution of the SIR epidemic model [Journal Article]. Physica D. 2020;408:132540.

33. Harko T, Lobo FSN, Mak MK. Exact analytical solutions of the susceptible-infected-recovered (SIR) epidemic model and of the 

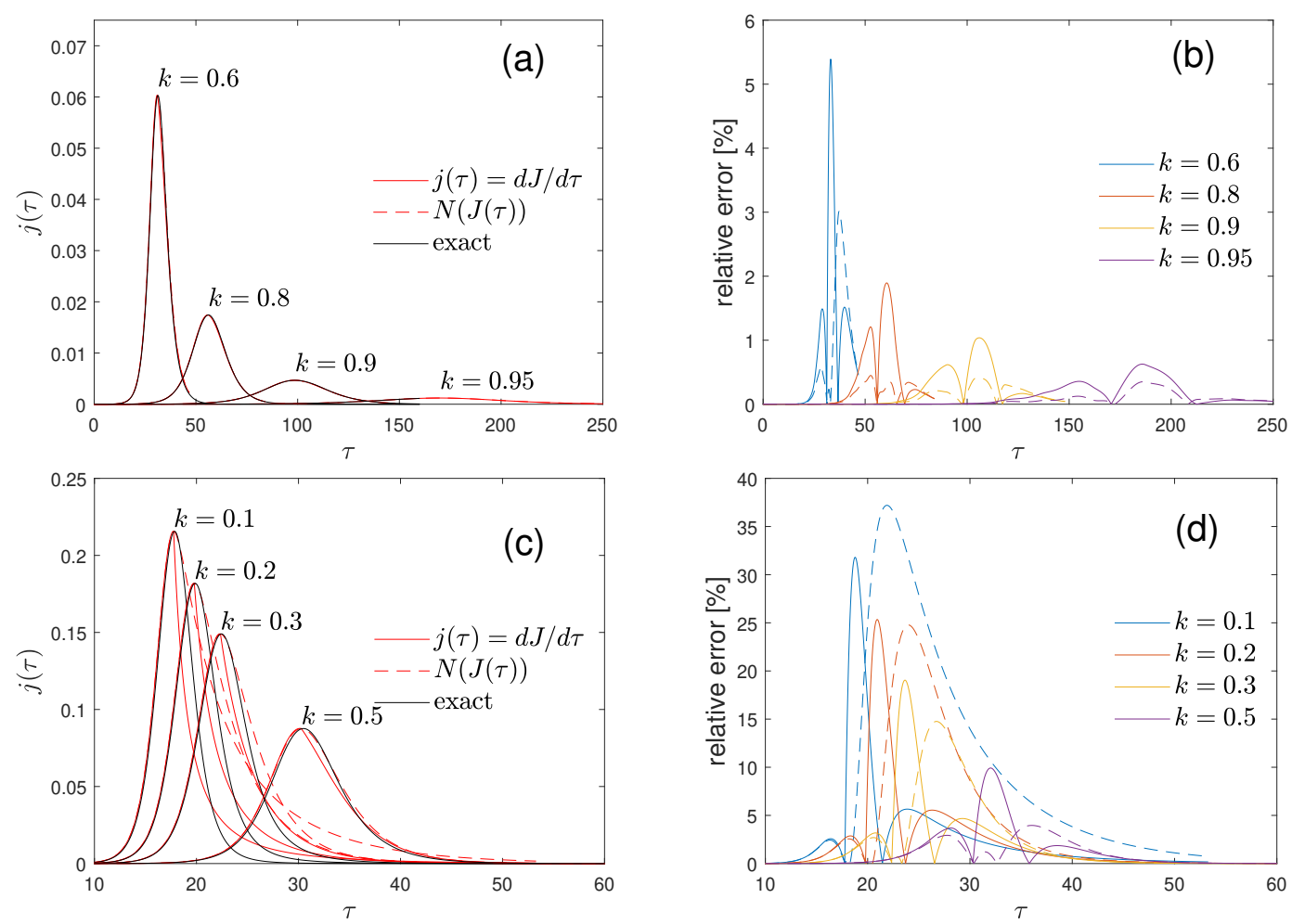

FIG. 5. (a,c) Comparison between exact and here developed approximate solutions to the semi-time SIR model, for various $k$. (b,d) Relative error of the approximants shown in (a,b): $j(\tau)$ from Eq. (23), which is identical with $d J / \tau$ and also identical with $j(\tau)$ according to Eq. (16) (solid) and $j(\tau)=N(J(\tau))$ according to Eq. (3), with $J(\tau)$ from Eq. 20. The plot is done with $\eta=10^{-6}$, but it is qualitatively unaffected by the choice of $\eta$, not only for small $\eta \ll 1$.

SIR model with equal death and birth rates. Appl Math Comput. 2014;236:184-194.

34. Kröger M, Schlickeiser R. Analytical solution of the SIRmodel for the temporal evolution of epidemics. Part A: Timeindependent reproduction factor. J Phys A: Math Theor. 2020;53:505601.

35. Schlickeiser R, Kröger M. Analytical solution of the SIR-model for the temporal evolution of epidemics. Part B: Semi-time case. Preprints. 2021. Submitted.
36. Kröger M, Schlickeiser R. Gaussian doubling times and reproduction factors of the Covid-19 pandemic disease. Frontiers Phys. 2020;8:276.

37. Komarova NL, Schang LM, Wodarz D. Patterns of the COVID19 pandemic spread around the world: exponential versus power laws. J R Soc Interface. 2020;17(170):20200518.

38. Park SW, Bolker BM, Champredon D, Earn DJD, Li M, Weitz JS, et al. Reconciling early-outbreak estimates of the basic reproductive number and its uncertainty: framework and applications to the novel coronavirus (SARS-CoV-2) outbreak. J R Soc Interface. 2020;17(168):20200144. 

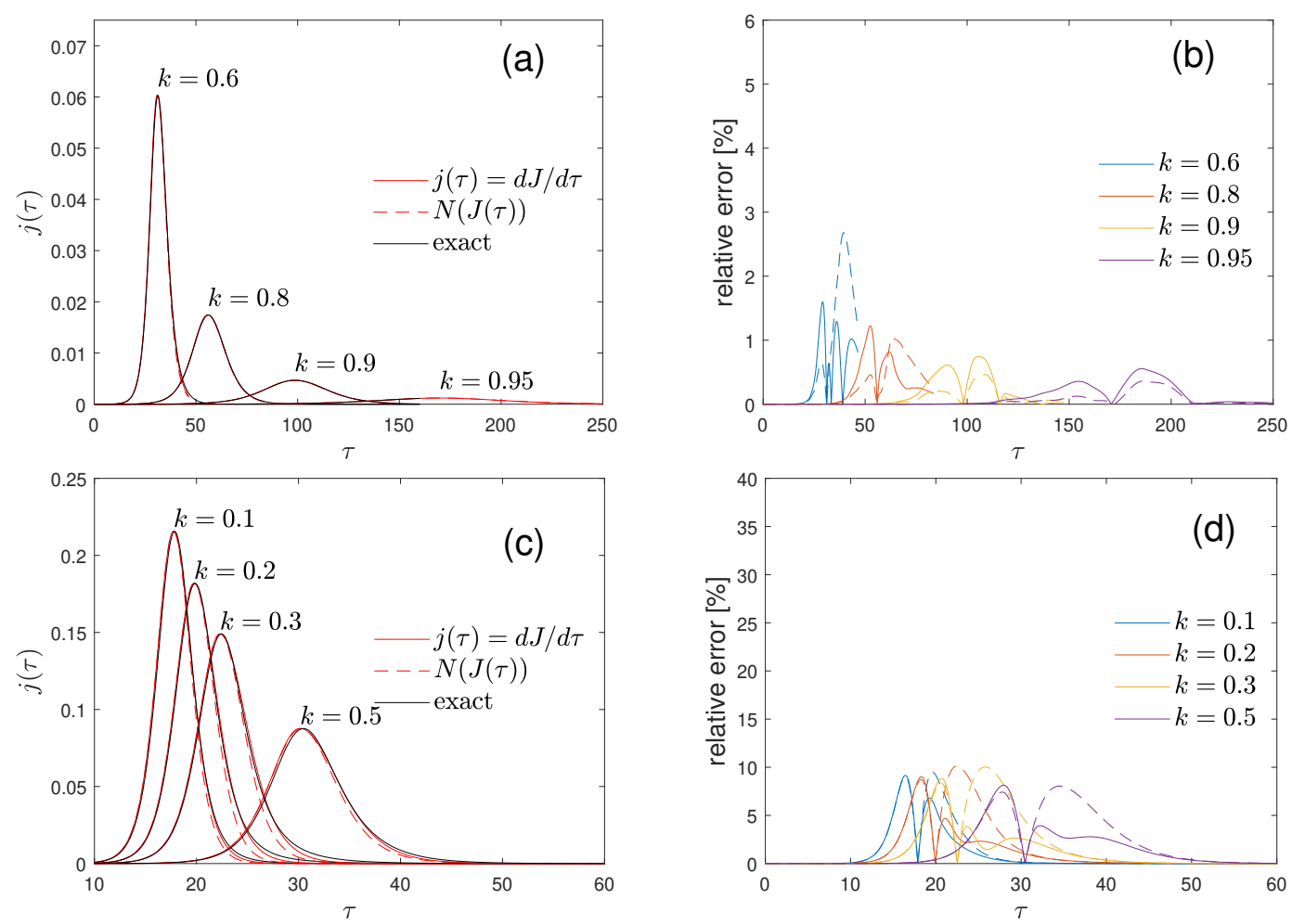

FIG. 6. (a,c) Comparison between exact and approximate solutions to the semi-time SIR model, denoted as version II in our earlier work, ${ }^{35}$ for various $k$. (b,d) Relative error of the approximants shown in (a,b): $j(\tau)$ from Eq. (23), which is identical with $d J / \tau$ and also identical with $j(\tau)$ according to Eq. (16) (solid) and $j(\tau)=N(J(\tau))$ according to Eq. (3), with $J(\tau)$ from Eq. 20. The plot is done with $\eta=10^{-6}$, but it is qualitatively unaffected by the choice of $\eta$, not only for small $\eta \ll 1$. While for small $k$ the error is significantly smaller for this approximant compared to the approximant used in the present work, the present approximant has the neat features that it exactly reaches the exact $j_{\text {max }}$ at peak time, and that it possesses the correct asymptotic behavior, which is not reflected by watching the relative error. Furthermore, the present approximant has a smaller error than the so-called version I approximant. ${ }^{35}$. 